Revue d'histoire de l'enfance « irrégulière »

Le Temps de l'histoire

1 | 1998

La protection de l'enfance : regards

\title{
Protection judiciaire de l'enfance en Algérie : Le secteur associatif (1945-1963)
}

\section{(2) OpenEdition \\ Journals}

Édition électronique

URL : http://journals.openedition.org/rhei/21

DOI : 10.4000/rhei.21

ISBN : 978-2-7535-1638-0

ISSN : $1777-540 \mathrm{X}$

Éditeur

Presses universitaires de Rennes

\section{Édition imprimée}

Date de publication : 15 novembre 1998

Pagination : 167-173

ISSN : 1287-2431

Référence électronique

Christian Sanchez, « Protection judiciaire de l'enfance en Algérie : Le secteur associatif (1945-1963) », Revue d'histoire de l'enfance « irrégulière » [En ligne], 1 | 1998, mis en ligne le 02 mai 2007, consulté le 04 décembre 2020. URL : http://journals.openedition.org/rhei/21 ; DOI : https://doi.org/10.4000/rhei.21 


\section{Protection judiciaire de l'enfance en Algérie}

\section{Le secteur associatif (1945-1963)}

Le rôle du secteur associatiffut important en Algérie ; nous avons demandé

à Christian Sanchez de réunir quelques informations sur ce secteur.

La vie sociale de l'Algérie de cette époque est singularisée par sa situation politique. ${ }^{(2)}$ Les notions de déviance et d'inadaptation lui deviennent propres. La pratique éducative en Algérie, pour le secteur considéré, est une pratique algérienne contextualisée par les données ethniques et la particularité administrative. Raymond Sultan, dans son article, évoque l'inertie de l'application des textes relatifs à l'enfance inadaptée.

Dès lors, les rapports d'activité, d'inspection, les témoignages recueillis sont à manier avec une extrême précaution. Tout au long du séminaire "l'Éducation surveillée, le secteur associatif en Algérie, 1945$1962 ",{ }^{(3)}$ sont apparues en permanence les blessures laissées par ce qui a été pour beaucoup une tragédie.

Sur le plan judiciaire, l'Algérie comprenait dix-sept arrondissements. La protection judiciaire de l'enfance inadaptée s'articulait autour d'un service public, l'Éducation surveillée, et d'un service privé habilité. Le docteur Maurice Porot ${ }^{(4)}$ précise que la délinquance double pendant la guerre, alors qu'elle quintuple en métropole pour la même période. La nature de la délinquance est sensiblement la même : «les garçons volent, les filles se prostituent, les uns les autres vagabondent $"{ }^{(5)}$

La population accueillie dans ces établissements en 1956, selon les mêmes sources, se répartissait ainsi :

\section{Christian \\ Sanchez ${ }^{(1)}$}

(1) Chef de service

éducatif à la Protection

judiciaire de la jeunesse.

(2) Une guerre sans

nom se développe sur son territoire depuis 1954.

(3) Un groupe de praticiens de l'enfance, ayant exercé ou non en Algérie, ont été réunis par l'Association pour l'histoire de l'éducation surveillée et de la protection judiciaire des mineurs, sous la direction d'une historienne, Françoise Tétard, pour tenter de retracer l'histoire de l'Éducation surveillée et du secteur associatif en Algérie. La restitution de ses travaux a été effectuée à

Vaucresson le 27 mai 1994, lors d'une journée organisée par le Service des études du CNFE et par l'AHES-PJM. 
(4) Président du

Comité algérien de liaison des activités en faveur de l'enfance délinquante.

(5) M. Porot, Revue de la Sauvegarde, n 8, 1950, p. 527.

(6) Association nationale des éducateurs de jeunes inadaptés. Les sources proviennent d'une enquête effectuée, en 1955, par cet organisme et sont citées par J. Gauneau, op. cit.

(7) Ceci concerne bien sûr les établissements privés.

\begin{tabular}{|c|c|c|c|c|}
\hline Secteur & Origine & Oran & Alger & Constantine \\
\hline \multirow{2}{*}{ Public } & Musulmans & 540 & 1.620 & 716 \\
& Européens & 110 & 380 & 45 \\
\hline \multirow{2}{*}{ Privé } & Musulmans & 120 & 1.220 & 90 \\
& Européens & 155 & 925 & 105 \\
\hline
\end{tabular}

\section{LE PERSONNEL ÉDUCATIF}

En 1955, l'encadrement éducatif était assuré par 320 personnes, dont 180 relevaient des associations. Le degré de spécialisation se répartissait ainsi :

\begin{tabular}{|c|c|c|c|c|c|}
\hline \multirow{2}{*}{$\begin{array}{c}\text { Origine } \\
\text { des éducateurs }\end{array}$} & \multicolumn{2}{|c|}{ Effectifs } & \multirow[t]{2}{*}{ Diplômés } & \multirow[t]{2}{*}{ Formés } & \multirow[t]{2}{*}{ Non formés } \\
\hline & Public & Privé & & & \\
\hline Musulmans & 1 & 35 & 1 & 6 & 29 \\
\hline Européens & 139 & 145 & 6 & 79 & 199 \\
\hline Total & 140 & 180 & 7 & 85 & 228 \\
\hline Ensemble & \multicolumn{2}{|c|}{320} & \multicolumn{3}{|c|}{320} \\
\hline
\end{tabular}

Ce tableau est établi en considérant comme formés les éducateurs ayant bénéficié d'une formation reconnue par les ministères et par l'ANEJI. ${ }^{(6)}$ Il n'existe pas à cette époque, en Algérie, d'école de formation d'éducateurs; c'est pour cette raison que Jacques Gauneau, en soulignant ce manque, demandait alors, au nom de l'ANEJI, que les établissements n'embauchent plus d'éducateurs si ces derniers n'étaient pas titulaires d'un diplôme de moniteur de colonie de vacances. ${ }^{(7)}$

En 1956, l'association "Moissons nouvelles" crée un centre de for- 
mation d'éducateurs de l'enfance inadaptée, qui recevra l'agrément du conseil technique le 9 janvier 1959. Ce centre allait former les travailleurs sociaux devant exercer dans «les établissements publics relevant des divers organismes de tutelle (Santé-Population et Aide sociale; éventuellement Éducation nationale et Éducation surveillée) ou dans des associations privées agréées par ces organismes $" .{ }^{(8)}$

Pour entrer dans ce centre de formation, aucun diplôme n'était en principe exigé. Mais le niveau du bac ou d'un brevet d'enseignement industriel était souhaité. Des dérogations pouvaient être accordées pour ne pas écarter les "candidats doués d'un sain équilibre psychologique, d'aptitudes pédagogiques réelles, d'une bonne intelligence, mais d'un niveau scolaire ne dépassant pas celui d'un BE ou d'un CAP. Plus qu'une culture scolaire élevée, le centre de formation requiert une ouverture d'esprit, un jugement sain, une curiosité en éveil ». ${ }^{(9)}$ Il est à noter que le centre invitait les candidats non détenteurs du diplôme de moniteur de colonie de vacances à suivre une session de formation à ce diplôme. La formation dispensée s'étalait sur trois ans; elle alternait stage pratique, la première année, acquisition des techniques de base et études théoriques, la seconde année, pour s'achever sur une troisième année de stage.

\section{LES ÉTABLISSEMENTS}

Cinq grandes associations du secteur associatif géraient les établissements répartis sur l'ensemble du territoire.

\section{1 - L'équipe sociale de préservation de l'enfance en danger moral}

Cette association était animée par le Docteur Porot et dirigée par le service social, avec la collaboration de psychiatres qui intervenaient à l'Éducation surveillée. Le personnel était principalement composé de bénévoles issus des écoles d'assistantes et d'étudiants ou de jeunes scouts. Le projet de cette association était la sauvegarde des enfants en situation de danger moral, comme le stipulait l'article 2 de ses statuts :

"Cette association a pour but de sauvegarder les enfants européens ou indigènes que leurs conditions de famille, de travail, mettent en danger moral : 
(10) Cité par le Dr M. Porot, op cit., p. 538.

(11) M. Porot, op. cit., p. 537.

(12) Juge musulman, dont la compétence s'étend aux questions en rapport avec la religion. $1^{\circ}$ en redonnant son importance au cadre familial, soit en l'étayant, soit en le rééduquant:

$2^{\circ}$ en donnant des distractions saines et adaptées à leur âge;

$3^{\circ}$ en aidant au placement en apprentissage de ceux qui ont dépassé l'âge scolaire, ou en leur permettant de compléter leur instruction générale ou professionnelle;

$4^{\circ}$ en intervenant, le cas échéant, auprès des autorités, pour provoquer l'application des mesures légales propres à la préservation de ces enfants et à la défense de leurs droits ». ${ }^{(10)}$

L'association disposait de deux structures à Alger :

\section{Le service social auprès du tribunal}

Son activité était multiple : "enquêtes sociales et médicales, interrogatoires des mineurs, constitution d'un dossier, organisation des examens médico-psychologiques et médicaux, surveillance des placements, préparation de la sortie des mineurs en liaison avec les services intéressés... " étaient notamment à sa charge. "Les rapports sont constants avec le tribunal, la police des mours, l'Assistance publique, les maisons d'observation et de rééducation, pour ne parler que des organismes officiels». ${ }^{(11)}$

\section{Le centre d'accueil d'Héliopolis}

Rattaché aux tribunaux pour enfants de Guelma, Bône et Philippeville, il avait une capacité de 35 places. On y « conduit une observation simple ». Les mineurs y séjournent trois mois environ et partagent leur temps entre classe, éducation physique, jardinage et cueillette des olives chez des propriétaires voisins. Ce centre ferme en 1959 ou 1960, car il se trouve dans une zone militairement exposée.

\section{Le foyer de la rue Salvator-Abella, à Bône}

Construit par les jeunes et l'équipe éducative, ce foyer peut accueillir 24 adolescents de 14 à 21 ans.

\section{2 - L'Aide et protection à l'enfance algérienne}

Cette association, créée en 1948, avait comme président le cadi ${ }^{(12)}$ d'Alger. S'occuper de l'enfance délaissée et délinquante était son objectif; pour 
cela elle disposait de deux établissements :

Le centre de Dalmatie, du nom du petit village où il était situé, à trois kilomètres de Blida.

Le centre de Mostaganem. Situé à Pellicier, à 3 kilomètres de Mostaganem, il occupait une ferme de dix hectares et comportait une section d'accueil et une section agricole pouvant accueillir 40 mineurs.

\section{3 - Les Bons Pasteurs}

Deux établissements, situés à $\boldsymbol{E l}$ Biar et Missergbin, recevaient cent vingt-cinq filles, séparées en deux sections : la première section œuvrait au rattrapage scolaire, la seconde visait à former de jeunes ménagères (cuisine, buanderie, repassage, etc.) et des sténodactylos.

Ces Bons Pasteurs fonctionnaient à l'instar de toutes les autres institutions de la congrégation-mère d'Angers.

\section{4 - L’Association pour la formation de la jeunesse}

Cette association, fondée en avril 1957 sous l'impulsion de la générale Massu, recevait des "jeunes adolescents de 14 à 17 ans qui se trouvaient après leur scolarité livrés à la rue et dangereusement exposés ». ${ }^{(13)}$ Elle gérait plusieurs établissements :

Le centre de jeunesse de Bab-el-Oued, pouvait recevoir 82 mineurs, orphelins et vagabonds;

Deux autres foyers, dits "foyers des yaouleds" ${ }^{(14)}$ respectivement d'une capacité de 45 et 20 mineurs, se trouvaient à Alger, l'un à proximité de la casbah, l'autre à côté du port.

5 - L'Association pour l'éducation professionnelle et familiale des jeunes (Moissons nouvelles)

Cette association, créée en 1941 sous l'impulsion de la JOC, ${ }^{(1)}$ se consacrait à l'éducation professionnelle et familiale des jeunes. Trois périodes sont à considérer pour cette association.

De 1941 à 1946, l'association va transformer les chantiers en structures nouvelles : 


\section{Le centre artisanal de Beau Fraisier}

Situé dans le quartier populaire de Bab-el-Oued. D'une capacité d'accueil de 60 lits, il recevait des adolescents en insertion professionnelle et leur assurait une formation théorique et pratique.

\section{Un service d'accueil de jour}

Créé par Jean Abbad, kabyle chrétien, il assurait aux jeunes travailleurs des loisirs, un soutien scolaire ou une alphabétisation.

\section{Les centres de formation familiale et ménagère de Bône et de Philippeville}

Ils recevaient des jeunes filles en fin de scolarité et leur enseignaient une formation familiale, technique et ménagère. 80 jeunes filles pouvaient être accueillies à Bône, 60 à Phillipeville.

Durant la période de croissance de cette association (1947-1956), apparaissent de nouveaux établissements :

Deux centres d'apprentissage, un dans le quartier de Belcourt à Alger et l'autre à Oran.

Le foyer de semi-liberté d'Hussein-Dey, à Alger.

Le foyer des jeunes d'Ilonier, à Alger, ouvert dans les locaux annexes de l'ancien orphelinat St-Vincent-de-Paul.

La cité de l'enfance d'Alger installée dans les locaux de l'orphelinat de St-Vincent-de-Paul.

Le centre d'accueil, d'orientation et d'éducation de Tlemcen, en Oranie.

Après l'indépendance, en 1962, l'association poursuivra un temps ses activités. Puis, progressivement, les cadres des établissements sont changés. Le 3 mai 1965, un décret du gouvernement algérien décide le transfert à l'État algérien de l'école d'éducateurs et des établissements de Delly-Ibrahim, d'Hussein-Dey et de Tlemcen.

Une page de l'histoire de la rééducation des adolescents en Algérie est définitivement tournée, celle née de la présence française. 


\section{Bibliographie}

Communication de GAUNEAU (J.), in Actes du congrès de l'AIEJ (Association internationale des éducateurs de jeunes inadaptés), Bruxelles, 1956.

KRIEF (E.) et BOURDON (G.), "L'enfant algérien devant la loi et la justice pénale", Revue de la sauvegarde de l'enfance, nº, août-octobre 1950.

MICHARD (H.), Rapport d'inspection, 20 décembre 1954, Arch. CNFEPJJ, Vaucresson.

POROT (M.) et ROUY-AMIOT (C.), "La délinquance juvénile en Algérie", Revue de la sauvegarde de l'enfance, n8, août-octobre 1950.

SUTTER (J.), "L'assistance à l'enfance inadaptée en Algérie", Revue de la sauvegarde de l'enfance, $\mathrm{n}^{\circ} 8$, août-octobre 1950.

VOIRIN (P.) et GAILLAC (H.), Rapport d'inspection au directeur de l'Éducation surveillée, 27 août 1959, Arch. CNFE-PJJ, Vaucresson. 